\title{
The Lateral Decubitus Body Position Might Improve the Safety of Ultrasound-Guided Supraclavicular Brachial Plexus Nerve Block
}

This article was published in the following Dove Press journal: Journal of Pain Research

\author{
Carl PC Chen (iD) \\ Chih-Chin Hsu (D) ${ }^{2}$ \\ Chih-Hsiu Cheng ${ }^{3}$ \\ Shu-Chun Huang (D) \\ Jean-Lon Chen' \\ Shin-Yi Lin'
}

'Department of Physical Medicine \& Rehabilitation, Chang Gung Memorial Hospital at Linkou and College of Medicine, Chang Gung University, Taoyuan City, Taiwan; ${ }^{2}$ Department of Physical Medicine \& Rehabilitation, Chang Gung Memorial Hospital at Keelung and College of Medicine, Chang Gung University, Taoyuan City, Taiwan; ${ }^{3}$ School of Physical Therapy and Graduate Institute of Rehabilitation Science, College of Medicine, Chang Gung University, Taoyuan City, Taiwan
Correspondence: Carl PC Chen Department of Physical Medicine \& Rehabilitation, Chang Gung Memorial Hospital at Linkou and College of Medicine, Chang Gung University, 5, Fu-Hsin Street, Guishan, Taoyuan 333, Taiwan

Tel +886-909396/96

$\mathrm{Fax}+886-3-3281320$

Email carlchendr@gmail.com
Background: To investigate whether body mass index (BMI) and changes in body positioning have any correlation with the distance from the pleura to the inferior trunk of the supraclavicular brachial plexus.

Patients and Methods: Twenty stroke patients with upper limb spasticity and complex regional pain syndrome were recruited for this study. Distance from the pleura to the inferior trunk was measured in the supine position, body turned sideways at $45^{\circ}$, and in the lateral decubitus position. Correlations between BMI and measured distances in these body positions were evaluated. A linear musculoskeletal ultrasound transducer was used to conduct these distance measurements.

Results: The distance from the pleura to the inferior trunk in the supine position was calculated to have an average of $0.42 \pm 0.06 \mathrm{~cm}$ (D1), $0.44 \pm 0.05$ (D2) when lying sideways at $45^{\circ}$, and $0.87 \pm 0.08 \mathrm{~cm}(\mathrm{D} 3)$ in the lateral decubitus position. The Kruskal-Wallis test revealed significant differences when comparing D3 with D1, and D3 with D2 $(p<0.001)$. Positive correlations were observed between BMI and D1 (Spearman's rho $=0.62, p=0.004$, two-tailed), and between BMI and D2 (Spearman's rho $=0.61, p=0.005$, two-tailed). The strongest positive correlation was observed between BMI and D3 (Spearman's rho $=0.78$, $p<0.001$, two-tailed).

Discussion: In the lateral decubitus body position, the distance from the pleura to the inferior trunk increased significantly by 2 -fold and was positively correlated with BMI. The increased distance may improve the safety of the nerve block procedure. As a result, it is recommended that patients be placed in the lateral decubitus body position when performing ultrasound-guided supraclavicular brachial plexus nerve block of the inferior trunk.

Keywords: body mass index, ultrasound-guided, brachial plexus, nerve block, inferior trunk, lateral decubitus, corner pocket, complex regional pain syndrome

\section{Introduction}

Supraclavicular brachial plexus nerve block can be performed to achieve effective anesthesia and postoperative analgesia of the upper limb. ${ }^{1}$ This procedure can also be used for the treatment of various upper limb disorders such as spasticity, neuropathic pain, and complex regional pain syndrome (CRPS). ${ }^{2,3}$ The aim of this block is the placement of an injection needle into the corner pocket, as practiced by most practitioners. The corner pocket is located between the first rib or the pleura inferiorly, the subclavian artery medially, and the inferior trunk of the 
supraclavicular brachial plexus superiorly. ${ }^{4}$ This approach is preferred for brachial plexus nerve block because of its ulnar sparing property. ${ }^{5}$ This procedure can be performed using the blind anatomical palpation method combined with the application of a nerve stimulator. ${ }^{6}$ Since musculoskeletal ultrasound can be used as an effective tool in guiding the needle to the designated injection target site, supraclavicular brachial plexus nerve block is often performed nowadays using ultrasound guidance with higher accuracy. $^{7}$

Conventionally, an ultrasound-guided supraclavicular brachial plexus nerve block is performed with the patient lying in a reclined supine position of approximately $30^{\circ}$, with a pillow under the head. A high-frequency linear transducer was used, and the block was performed in an inplane, lateral-to-medial approach by inserting the needle into the corner pocket. Although some practitioners may also perform a subsequent ultrasound-guided block of the proximal trunks of the brachial plexus, this study only examined the procedure of ultrasound-guided nerve block to the inferior trunk. Even if the block is performed meticulously using ultrasound guidance, delayed pneumothorax can still occur. ${ }^{7,8}$ The complication of pneumothorax might be due to the short distance from the pleura to the inferior trunk. Studies that aim to improve the safety of ultrasoundguided supraclavicular brachial plexus block with the adjustment in body positioning are limited. ${ }^{9}$

The traction force of the brachial plexus can be affected by the human body posture. Factors such as the degrees of spine retroflexion, lateroflexion, and rotational movements of the humerus can affect the traction force of the brachial plexus. ${ }^{9}$ Body mass index (BMI) can influence the depth of the corner pocket. It was reported that BMI is positively correlated with the depth of the corner pocket when measured from the skin. ${ }^{4}$ In other words, as BMI increases, the distance from the skin to the corner pocket increases. In this study, stroke patients with upper limb spasticity and CRPS were recruited for ultrasound-guided supraclavicular brachial plexus nerve block. The distance between the pleura and the inferior trunk of the supraclavicular brachial plexus was measured with the patients in the supine position, turned sideways at $45^{\circ}$, and in the lateral decubitus position. The BMI of these patients was also calculated and further correlated with the measured distances in these three different body positions. We hypothesized that BMI is positively correlated with the distance from the pleura to the inferior trunk, and that this distance is the longest in the lateral decubitus body position. Therefore, the lateral decubitus body position can be the preferred body position when performing ultrasound-guided supraclavicular brachial plexus nerve block. With the increased distance from the pleura to the brachial plexus, the complication of pneumothorax might be reduced.

\section{Patients and Methods}

\section{Patients}

This was a prospective study. A total of 20 patients with a diagnosis of stroke combined with spasticity and CRPS of the upper limb were recruited. The aim was to perform a successful ultrasound-guided supraclavicular brachial plexus nerve block of the inferior trunk by injecting the anesthetic agent into the corner pocket. This study was approved by the Institutional Review Board (IRB) of Chang Gung Medical Foundation and was conducted in accordance with the Declaration of Helsinki. All patients signed written informed consent before participating in this study (IRB approval number: 202001039A3). Patients with severe spasticity of the neck and trapezius muscle, resulting in difficulties in the placement of the ultrasound transducer and exposure of the needle insertion site were excluded from the study.

\section{Measurements}

Distance Between the Pleura and the Inferior Trunk Under 3 Body Positions

This distance was measured in 3 different body positions using the BenQ T3300 Tablet Ultrasound System with L154BH Linear Array transducer (frequency of 4-15 $\mathrm{MHz}$ ) (BenQ Corporation, Taiwan).

\section{Position I}

Patients lying in a supine position reclined at approximately $30^{\circ}$. A pillow was placed under the head, with the head turned away from the examination site for maximal exposure of the ultrasound examination area. The anatomical location of the brachial plexus, subclavian artery, and scalene muscles are shown in Figure 1. The measured distance from the pleura to the inferior trunk of the supraclavicular brachial plexus in this position is shown as D1 (Table 1). The technique of ultrasoundguided placement of the injection needle into the corner pocket is shown in Figure 2.

\section{Position 2}

Patients lying sideways at approximately $45^{\circ}$. Pillows are placed under the head and under the back to ensure that patients can remain in this body position comfortably. The 


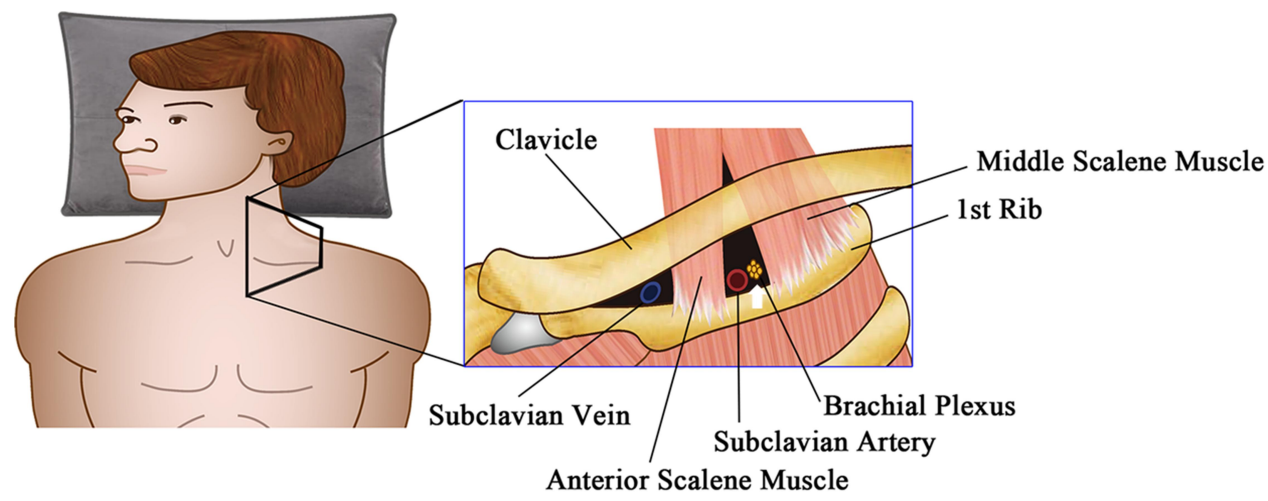

Figure I Anatomical position of the supraclavicular brachial plexus and its relationship with the subclavian artery and the scalene muscles. The patient was placed in the supine position, and the head turned away from the ultrasound examination site. White arrow indicates the location of the corner pocket.

measured distance from the pleura to the inferior trunk of the supraclavicular brachial plexus in this position is shown as D2 (Table 1).

\section{Position 3}

Patients in lateral decubitus body position. In this position, the ipsilateral upper limb is rested comfortably in front of the chest wall. The shoulder joint was adducted, and the humerus was internally rotated (Figure 3). The measured distance from the

Table I Patient Characteristics and Measured Variables

\begin{tabular}{|c|c|c|c|c|c|c|}
\hline Patient Number & Sex & Age (y) & BMI & DI & D2 & D3 \\
\hline I & $\mathrm{F}$ & $4 I$ & 22.3 & 0.34 & 0.36 & 0.78 \\
\hline 2 & $M$ & 56 & 23.4 & 0.47 & 0.5 & 0.88 \\
\hline 3 & $M$ & 51 & 21.3 & 0.41 & 0.47 & 0.76 \\
\hline 4 & $\mathrm{~F}$ & 47 & 22.5 & 0.45 & 0.41 & 0.91 \\
\hline 5 & $M$ & 62 & 21.7 & 0.36 & 0.43 & 0.81 \\
\hline 6 & $F$ & 63 & 24.8 & 0.49 & 0.44 & 0.97 \\
\hline 7 & $\mathrm{~F}$ & 61 & 26.7 & 0.42 & 0.51 & I.II \\
\hline 8 & $M$ & 55 & 23.6 & 0.38 & 0.43 & 0.95 \\
\hline 9 & $\mathrm{~F}$ & 57 & 21.4 & 0.33 & 0.36 & 0.81 \\
\hline 10 & $M$ & 59 & 23.7 & 0.46 & 0.48 & 0.92 \\
\hline II & M & 46 & 21.5 & 0.4 & 0.45 & 0.86 \\
\hline 12 & $\mathrm{~F}$ & 36 & 19.9 & 0.37 & 0.42 & 0.83 \\
\hline 13 & M & 56 & 21.6 & 0.29 & 0.33 & 0.77 \\
\hline 14 & $M$ & 60 & 24.1 & 0.43 & 0.49 & 0.93 \\
\hline 15 & $M$ & 65 & 25.7 & 0.51 & 0.47 & 0.89 \\
\hline 16 & $M$ & 68 & 26.9 & 0.49 & 0.54 & 0.94 \\
\hline 17 & $\mathrm{~F}$ & 48 & 21.4 & 0.44 & 0.41 & 0.87 \\
\hline 18 & $M$ & 55 & 21.6 & 0.38 & 0.44 & 0.79 \\
\hline 19 & $F$ & 65 & 22.1 & 0.48 & 0.39 & 0.81 \\
\hline 20 & $M$ & 54 & 23.8 & 0.42 & 0.46 & 0.9 \\
\hline
\end{tabular}

Notes: DI: Distance from the pleura to the supraclavicular brachial plexus inferio trunk in centimeters. Patient under the supine position. D2: Distance from the pleura to the supraclavicular brachial plexus inferior trunk in centimeters. Patient lies sideways at about 45 degrees. D3: Distance from the pleura to the supraclavicular brachial plexus inferior trunk in centimeters. Patient under the lateral decubitus position. Abbreviations: F, female; $M$, male; $y$, age in years. pleura to the inferior trunk of the supraclavicular brachial plexus in this position is shown as D3 (Table 1). The technique of ultrasound-guided placement of the injection needle into the corner pocket in this position is shown in Figure 3.

\section{$\mathrm{BMI}$}

BMI was calculated for all the recruited patients using the following formula: Weight (in kilograms $[\mathrm{kg}] / \mathrm{Height}^{2}$ (in meters squared $\left.\left(\mathrm{m}^{2}\right)\right)$ ).

According to the World Health Organization BMI categorization in Asian populations, patients with a BMI of 23-24.9 are considered mildly overweight, and patients with a BMI of 25-27.4 are considered pre-obese. ${ }^{10}$

\section{The Supraclavicular Brachial Plexus Nerve Block}

After strict sterilization, the injection needle was inserted under the in-plane, and in a lateral-to-medial approach using musculoskeletal ultrasound guidance. The needle was inserted into the corner pocket, with the aim of bathing the brachial plexus inferior trunk with an adequate amount of anesthetic. The local anesthetic solution of $1 \%$ lidocaine was used. The purpose of the block was to alleviate the upper limb pain of the recruited patients, caused by CRPS. Before the injection procedure, color Doppler examination should be performed to ensure that the needle does not hit any neurovascular structures during the nerve block procedure. In this study, the distance measurements, the block procedure, and the interpretation of the ultrasound images were performed by the same physiatrist to prevent experimental bias.

\section{Statistical Analysis}

The distance from the pleura to the supraclavicular brachial plexus was analyzed using the Kruskal-Wallis non-parametric 


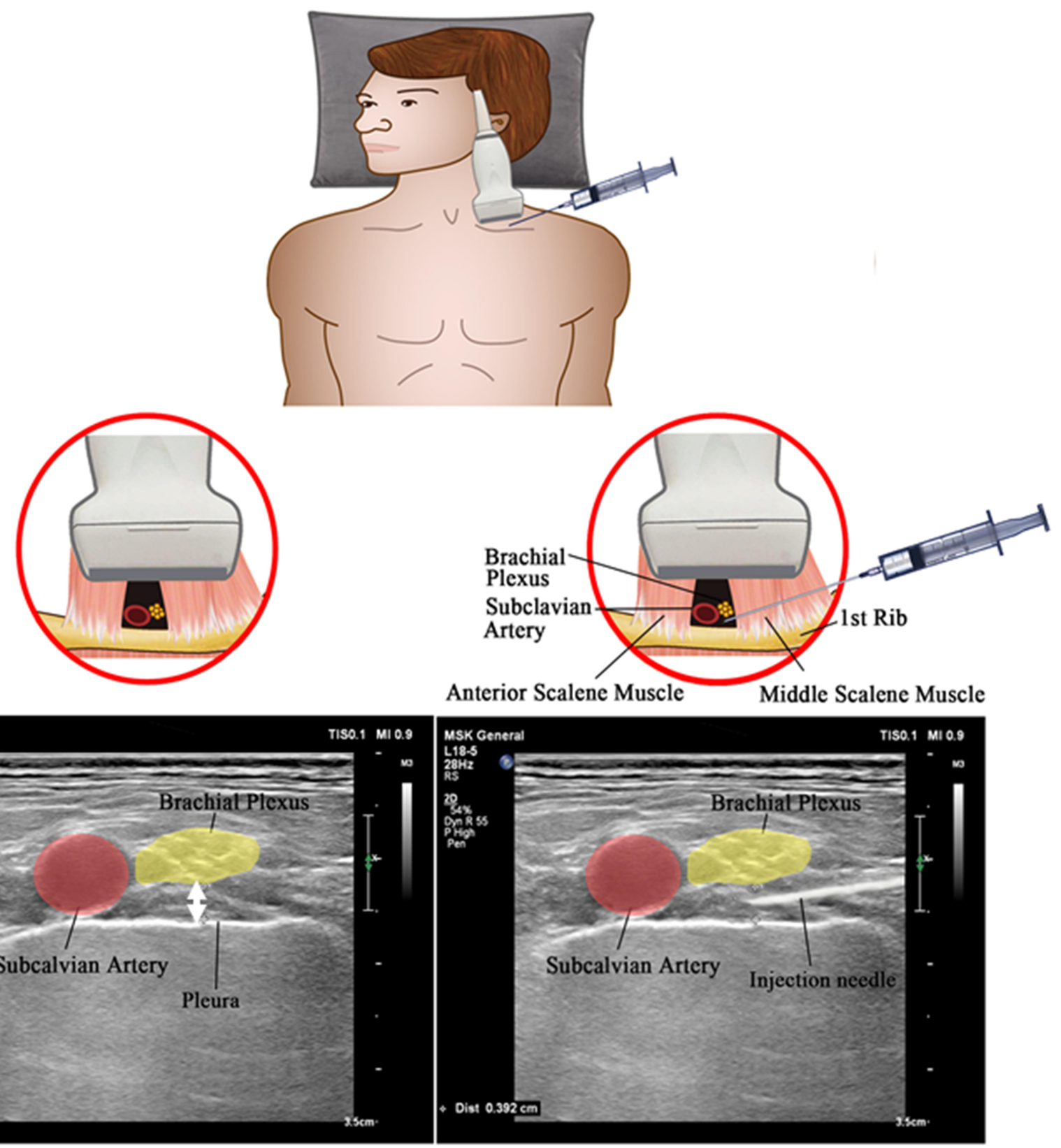

Figure 2 In the supine position, the head is turned away from the side of nerve block for maximum exposure of the brachial plexus. The brachial plexus and the subclavian artery are located at about the same height. The double white arrow indicates the distance from the pleura to the inferior trunk of the brachial plexus, which is about $0.4 \mathrm{~cm}$.

analysis of variance (ANOVA) followed by post hoc Dunn's test for multiple comparisons. The non-parametric Spearman's rank-order correlation coefficient was used to analyze the correlations between BMI and the measured distances of D1, D2, and D3. In addition, scatter plots were plotted to show the correlations between BMI and these distances. Each plot was also fitted with a linear trend line. Data are expressed as mean \pm standard deviation and were analyzed using SPSS 21 (SPSS version 21.0, SPSS Inc., United States). A $p$-value of less than 0.05 was considered statistically significant. The sample size was set at 20 because a significant difference was achieved using the Kruskal-Wallis non-parametric ANOVA statistical analytical tool.

\section{Results}

BMI, sex, age, and the distances measured from the pleura to the supraclavicular brachial plexus inferior trunk in three different body positions for the recruited patients (12 men, 8 women) are listed in Table 1 . The mean age of the male patients was $57 \pm 6.0$ years (range, 46-68 

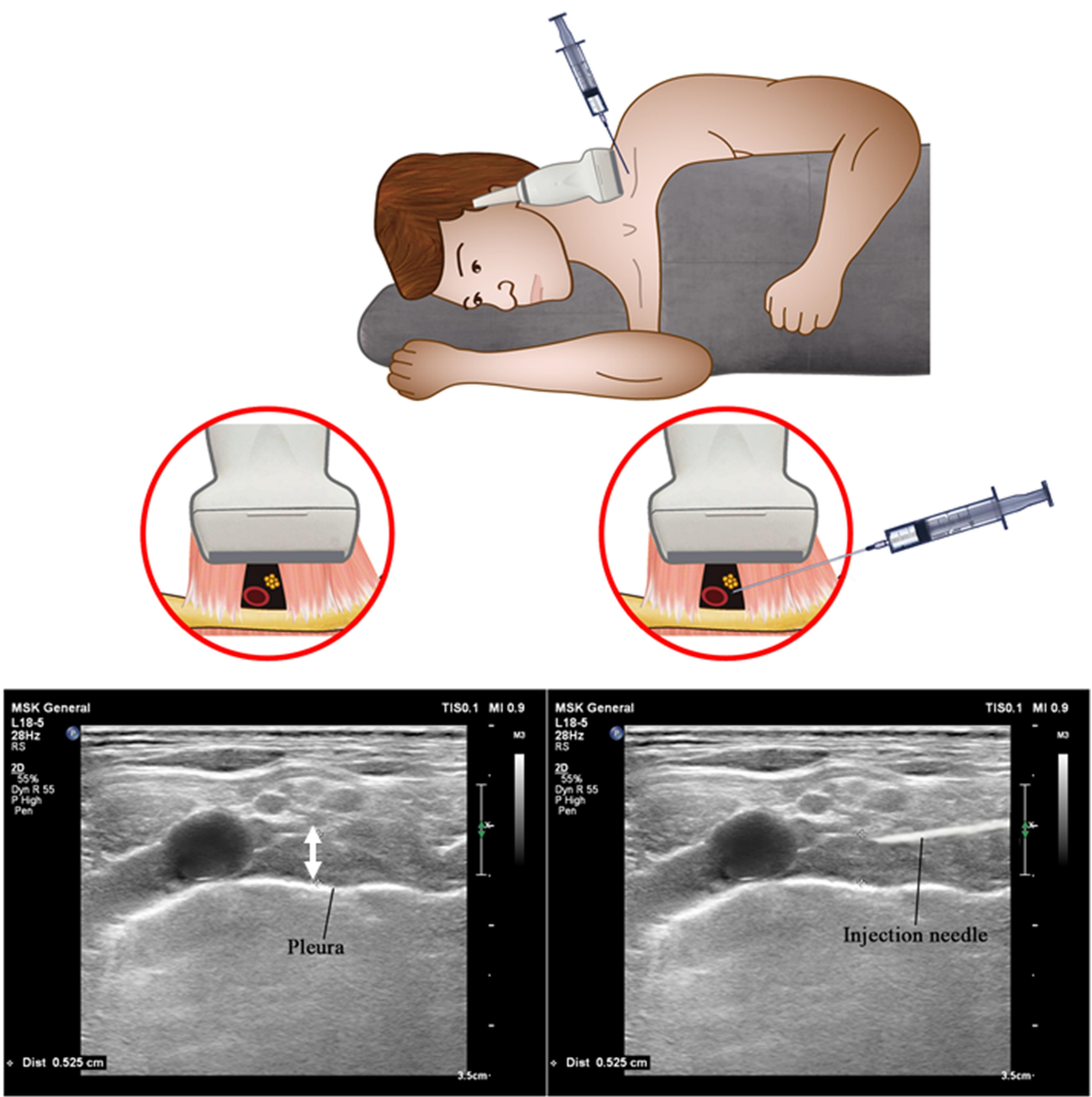

Figure 3 In the lateral decubitus position, the head is well rested on the pillow. A pillow is also placed in front of the chest wall, with the upper limb comfortably placed on the pillow. The double white arrow indicates significant increase in the distance from the pleura to the inferior trunk. Sonographic assessment showed that the brachial plexus has moved proximally in relation to the subclavian artery.

years). The mean age of the female patients was $52 \pm 10.8$ years (range, 36-65 years). The mean BMI for the male patients was $23.2 \pm 1.8 \mathrm{~kg} / \mathrm{m}^{2}$ (range, $21.3-26.9 \mathrm{~kg} / \mathrm{m}^{2}$ ), and the mean BMI for the female patients was $22.6 \pm$ $2.1 \mathrm{~kg} / \mathrm{m}^{2}$ (range, $19.9-26.7 \mathrm{~kg} / \mathrm{m}^{2}$ ). The overall mean BMI was $23.0 \pm 1.9 \mathrm{~kg} / \mathrm{m}^{2}$.

In the supine position, the distance from the pleura to the supraclavicular brachial plexus inferior trunk (D1) was calculated to have an average of $0.42 \pm 0.06 \mathrm{~cm}$. This distance was calculated to be $0.44 \pm 0.05 \mathrm{~cm}$ in the sideways lying position at $45^{\circ}$ (D2). In the lateral decubitus position, this distance was calculated to be $0.87 \pm 0.08 \mathrm{~cm}$ (D3). The Kruskal-Wallis test revealed significant differences between D1 and D3, and between D2 and D3 $(p<$ 0.001). Statistical comparisons between D1 and D2 did not reveal any significant differences. The increase in the distance of D3 was twice that of D1 or D2 (Figure 4). Ultrasound images of the brachial plexus and the distance 


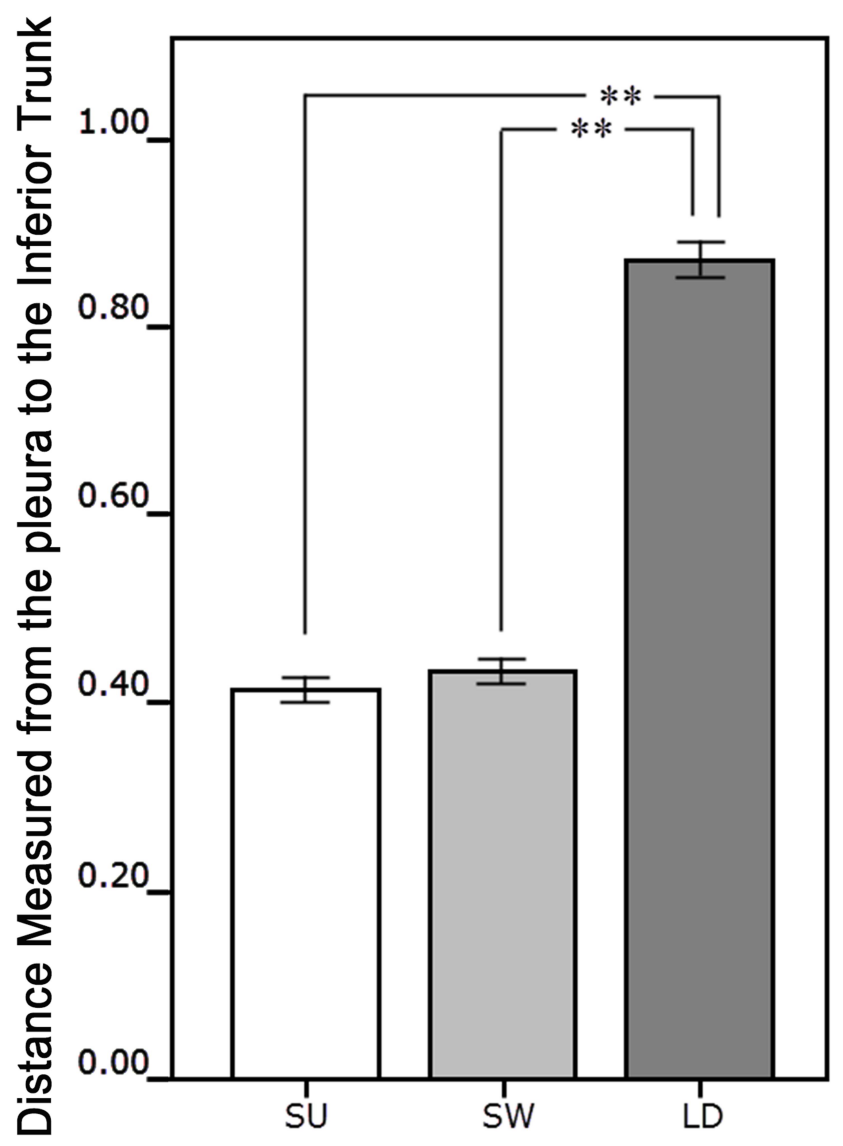

Figure 4 Average distance from the pleura to the brachial plexus inferior trunk expressed as mean \pm standard error of the mean. The average distance increased significantly by 2 -folds with the patients placed in the lateral decubitus body position. ${ }^{* * p}<0.00$ I, Kruskal-Wallis test followed by Dunn's test.

Abbreviations: SU, supine body position; SW, sideways at $45^{\circ}$; LD, lateral decubitus body position.

from the pleura to the supraclavicular brachial plexus inferior trunk revealed similar findings between the patients in the supine and $45^{\circ}$ sideways body positions.

Correlation analysis was performed between BMI and the measured distances in the three different body positions. Significant positive correlations were observed between BMI and D1 (Spearman's rho $=0.62, p=0.004$, twotailed), and between BMI and D2 (Spearman's rho $=0.61$, $p=0.005$, two-tailed). The strongest positive correlation was observed between BMI and D3 (Spearman's rho $=0.78$, $p<0.001$, two-tailed) (Figure 5).

\section{Discussion}

Supraclavicular brachial plexus nerve block is a reliable and predictable procedure performed to anesthetize the upper limb prior to surgeries. ${ }^{11}$ It is performed by inserting the needle into the corner pocket with the aim of bathing the supraclavicular brachial plexus inferior trunk with an

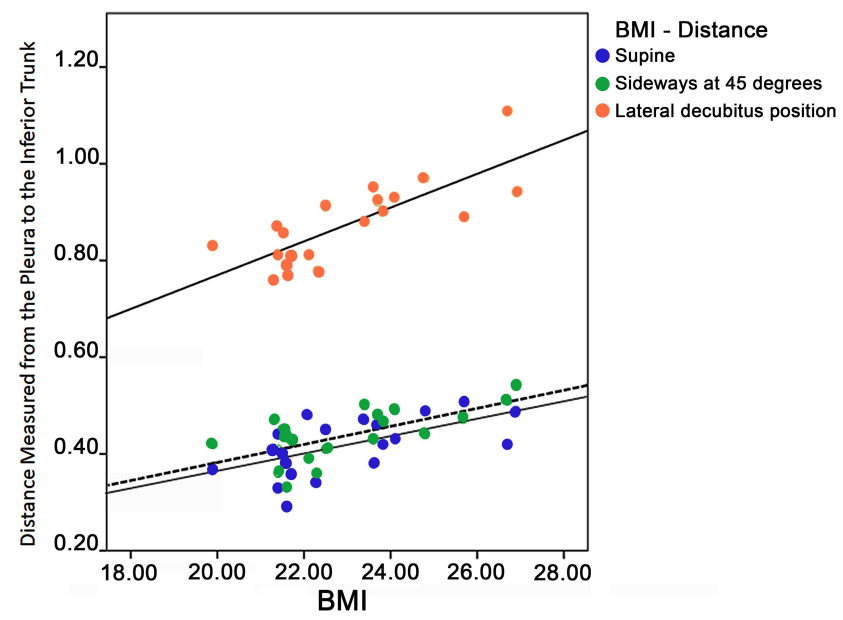

Figure 5 Scatter plots of BMI versus the distances from the pleura to the inferior trunk in three different body positions. Each plot was fitted with a linear trend line. Strongest positive correlation was observed with the body in the lateral decubitus position (Spearman's rho $=0.78, p<0.001$, two-tailed). Dashed linear trend line indicates positive correlation in sideways at $45^{\circ}$ body position.

adequate anesthetic. This is the area in which the brachial plexus is most compact. As a result, supraclavicular nerve blockade can provide fast and complete anesthesia of the upper limb. As practiced by most physicians, this procedure is commonly performed with patients in the supine position (Figure 1). It can be performed using the blind technique or under nerve stimulator guidance. The needle is inserted at the junction between the superior edge of the clavicle and the most lateral side of the sternocleidomastoid (SCM) muscle. ${ }^{12}$ Although the application of a nerve stimulator can assist in providing effective blocks, the risk of complications remains high. Common complications include pneumothorax, phrenic nerve paralysis, puncture of the vascular tissues, and even Horner syndrome. ${ }^{13,14}$ These complications did not occur in our recruited patients who received ultrasound-guided supraclavicular brachial plexus nerve block to the inferior trunk in the lateral decubitus body position. Even though the complication of nerve damage is usually self-limiting, precautionary measures should be taken.

Musculoskeletal ultrasound is an effective tool for guiding the needle to the required target site for nerve blocks. ${ }^{15}$ As a result, ultrasound is being widely used for supraclavicular brachial plexus nerve blocks. Chan et al are known to popularize this technique, with the use of a $7.5 \mathrm{MHz}$ transduce oriented $3 \mathrm{~cm}$ sagittally above the midpoint of the clavicle, with a success rate of up to $95 \% .{ }^{1}$ A study has documented that ultrasound guidance can speed the execution and quality of supraclavicular brachial plexus blocks. ${ }^{16}$ Without ultrasound guidance, a long 
learning curve is required to perform the block safely. To obtain a reasonable degree of proficiency, it is estimated that a physician needs to practice at least 62 blocks to perform this technique with a success rate of $87 \%$ without ultrasound guidance. ${ }^{17}$ Although ultrasound guidance has proven to be effective in improving the safety of supraclavicular brachial plexus block, complications such as pneumothorax can still occur. ${ }^{7,18}$ The logical cause of this complication may be due to the short distance between the pleura and the inferior trunk of the supraclavicular brachial plexus. For the conventional supine position nerve block, this distance was measured to be short, with an average of $0.42 \pm 0.06 \mathrm{~cm}$ (Figure 2). Therefore, even under extreme caution, it is still possible that the needle tip may accidentally penetrate into the pleura. Despite these complications, the supraclavicular brachial plexus nerve block is still preferred because of its ulnar sparing property and quick anesthesia onset time. ${ }^{5,16}$

This study examined the influence of body positioning on the distance from the pleura to the inferior trunk. The distance was approximately the same with the patient lying supine $(0.42 \pm 0.06 \mathrm{~cm})$ and sideways at $45^{\circ}(0.44$ $\pm 0.05 \mathrm{~cm})$. However, when patients were placed in the lateral decubitus position, sonographic assessment revealed that the supraclavicular brachial plexus moved proximally in relation to the subclavian artery, increasing this distance significantly by 2 -folds to $0.87 \pm 0.08 \mathrm{~cm}$. A logical explanation would be that in the lateral decubitus position, the upper limb on the nerve block side was well rested in front of the chest wall, with the shoulder adducted and the humeral head internally rotated (Figure 3 ). Together with the influence of gravity, the strain on the brachial plexus might have reduced, contributing to the upward sliding of the brachial plexus. Mihara et al have stated that certain rotations of the spine and shoulder placement angles will influence the strain and traction forces on the brachial plexus. ${ }^{9}$

A previous study examined the influence of BMI on the distance from the skin to the corner pocket. ${ }^{4}$ The authors concluded that BMI was positively correlated with this distance, and pre-scanning of the suprascapular region was recommended to estimate the depth of the corner pocket to select the appropriate needle size. ${ }^{4}$ Our study looked at the influence of BMI from a different perspective. Results showed positive correlations between $\mathrm{BMI}$ and the distances from the pleura to the inferior trunk. The strongest positive correlation was observed between BMI and the measured distance under the lateral decubitus body position. These results suggest that it is logical to place the patients under the lateral decubitus body position when performing ultrasound-guided supraclavicular brachial plexus nerve block. Of course, one may question whether it is difficult to perform the block in such a body position. In fact, we found that ultrasound-guided supraclavicular brachial plexus nerve block can be performed at ease in this body position, and there was no reported complication of pneumothorax post the nerve after performing the nerve block procedure. In this body position, the neck was well fixed on the pillow, and there was little or no pain retraction movements when the needle was inserted through the skin.

There were limitations to this study. The first limitation was the small number of recruited patients $(n=20)$. In future studies, more patients will be recruited and further analyses of BMI and its correlations with the distances from the pleura to the supraclavicular brachial plexus under different body positions will be conducted. The second limitation is that the recruited patients in this study did not have extremely high BMI values that indicated obesity (eg, BMI > 30). Whether patients with a higher BMI (possibly indicating thicker adipose tissue under the skin) will have significant positive correlations with the measured distances needs further validation. We will also examine the feasibility of performing ultrasoundguided supraclavicular nerve block by placing the needle tip deep into the brachial plexus sheath in the lateral decubitus body position to determine whether prolonged analgesia can be achieved. ${ }^{19}$

\section{Conclusions}

Musculoskeletal ultrasound is an effective tool to guide injection needles toward the required target sites to perform nerve blocks accurately. Ultrasound guidance is now being widely applied during supraclavicular brachial plexus nerve block as it has been proven superior to blind palpation and nerve stimulator guided injection methods. However, even with ultrasound guidance, the complication of pneumothorax may still occur. This is due to the short distance from the pleura to the inferior trunk of the supraclavicular brachial plexus, especially when patients are in the supine position. As a result, needle insertion into the corner pocket may cause accidental puncture of the pleura even when the nerve block procedure is performed meticulously under ultrasound guidance. The results of this study indicated that BMI is positively correlated with the distance from the pleura to the inferior trunk, and this distance is increased 
significantly by 2 -fold in the lateral decubitus body position. As a result, it is recommended that patients be placed in the lateral decubitus position when performing ultrasound-guided supraclavicular brachial plexus nerve block into the corner pocket using the in-plane and lateral-tomedial needle approach.

\section{Funding}

Support was provided solely from institutional and/or departmental sources.

\section{Disclosure}

The authors report no conflicts of interest in this work.

\section{References}

1. Chan VW, Perlas A, Rawson R, Odukoya O. Ultrasound-guided supraclavicular brachial plexus block. Anesth Analg. 2003;97 (5):1514-1517. doi:10.1213/01.ane.0000062519.61520.14

2. Fallatah SM. Successful management of complex regional pain syndrome type 1 using single injection interscalene brachial plexus block. Saudi J Anaesth. 2014;8(4):559-561. doi:10.4103/1658-354x.140903

3. Moon YE, Choi JH, Park HJ, Park JH, Kim JH. Ultrasound-guided nerve block with botulinum toxin type A for intractable neuropathic pain. Toxins (Basel). 2016;8(1):18. doi:10.3390/toxins8010018

4. Yadav N, Ayub A, Garg R, Nanda S, Gupta B, Sawhney C. Sonographic assessment of predictors of depth of the corner pocket for ultrasound-guided supraclavicular brachial plexus block. J Anaesthesiol Clin Pharmacol. 2016;32(1):25-28. doi:10.4103/ 0970-9185.175665

5. Dhir S, Brown B, Mack P, Bureau Y, Yu J, Ross D. Infraclavicular and supraclavicular approaches to brachial plexus for ambulatory elbow surgery: a randomized controlled observer-blinded trial. J Clin Anesth. 2018;48:67-72. doi:10.1016/j.jclinane.2018.05.005

6. Haleem S, Siddiqui AK, Mowafi HA, Ismail SA, Ali QA. Brief reports: nerve stimulator evoked motor response predicting a successful supraclavicular brachial plexus block. Anesth Analg. 2010;110(6):1745-1746. doi:10.1213/ANE.0b013e3181da82ff

7. Gupta K, Bhandari S, Singhal D, Bhatia PS. Pneumothorax following ultrasound guided supraclavicular brachial plexus block. J Anaesthesiol Clin Pharmacol. 2012;28(4):543-544. doi:10.4103/ 0970-9185.101962
8. Singh H. Pneumothorax following ultrasound guided supraclavicular brachial plexus block. J Anaesthesiol Clin Pharmacol. 2013;29 (3):411-412. doi:10.4103/0970-9185.117071

9. Mihara A, Kanchiku T, Nishida N, et al. Biomechanical analysis of brachial plexus injury: availability of three-dimensional finite element model of the brachial plexus. Exp Ther Med. 2018;15 (2):1989-1993. doi:10.3892/etm.2017.5607

10. Sauvaget C, Ramadas K, Thomas G, Vinoda J, Thara S, Sankaranarayanan R. Body mass index, weight change and mortality risk in a prospective study in India. Int $J$ Epidemiol. 2008;37 (5):990-1004. doi:10.1093/ije/dyn059

11. Lanz E, Theiss D, Jankovic D. The extent of blockade following various techniques of brachial plexus block. Anesth Analg. 1983;62 (1):55-58. doi:10.1213/00000539-198301000-00009

12. Hanumanthaiah D, Vaidiyanathan S, Garstka M, Szucs S, Iohom G. Ultrasound guided supraclavicular block. Med Ultrason. 2013;15 (3):224-229. doi:10.11152/mu.2013.2066.153.dh1mg2

13. Riegler FX. Brachial plexus block with the nerve stimulator: motor response characteristics at three sites. Reg Anesth. 1992;17 (5):295-299.

14. Ueshima H, Otake H. Incidence of phrenic nerve paralysis after ultrasound-guided supraclavicular brachial plexus block. $J$ Clin Anesth. 2019;56:37-38. doi:10.1016/j.jclinane.2019.01.030

15. Chen CPC, Chen JL, Ho CS, Suputtitada A. Ultrasound-guided medial branch blocks, facet joint, and multifidus muscle injections: how it is done under one needle insertion point! Anesthesiology. 2020;132(3):582-583. doi:10.1097/aln.0000000000003043

16. Honnannavar KA, Mudakanagoudar MS. Comparison between conventional and ultrasound-guided supraclavicular brachial plexus block in upper limb surgeries. Anesth Essays Res. 2017;11 (2):467-471. doi:10.4103/aer.AER_43_17

17. Duncan M, Shetti AN, Tripathy DK, Roshansingh D, Krishnaveni N. A comparative study of nerve stimulator versus ultrasound-guided supraclavicular brachial plexus block. Anesth Essays Res. 2013;7 (3):359-364. doi:10.4103/0259-1162.123235

18. Abell DJ, Barrington MJ. Pneumothorax after ultrasound-guided supraclavicular block: presenting features, risk, and related training. Reg Anesth Pain Med. 2014;39(2):164-167. doi:10.1097/ aap.0000000000000045

19. Sivashanmugam T, Ray S, Ravishankar M, Jaya V, Selvam E, Karmakar MK. Randomized comparison of extrafascial versus subfascial injection of local anesthetic during ultrasound-guided supraclavicular brachial plexus block. Reg Anesth Pain Med. 2015;40 (4):337-343. doi:10.1097/aap.0000000000000264
Journal of Pain Research

\section{Publish your work in this journal}

The Journal of Pain Research is an international, peer reviewed, open access, online journal that welcomes laboratory and clinical findings in the fields of pain research and the prevention and management of pain. Original research, reviews, symposium reports, hypothesis formation and commentaries are all considered for publication. The manuscript management system is completely online and includes a very quick and fair peer-review system, which is all easy to use. Visit http:// www.dovepress.com/testimonials.php to read real quotes from published authors. 